PROCEEDINGS OF THE

AMERICAN MATHEMATICAL SOCIETY

Volume 137, Number 5, May 2009, Pages 1579-1584

S 0002-9939(08)09684-6

Article electronically published on November 18,2008

\title{
THE FOLSOM-ONO GRID CONTAINS ONLY INTEGERS
}

\author{
SANDER ZWEGERS
}

(Communicated by Ken Ono)

\begin{abstract}
In a recent paper, Folsom and Ono constructed a canonical sequence of weight $1 / 2$ mock theta functions and a canonical sequence of weight $3 / 2$ weakly holomorphic modular forms, both using Poincaré series. They show a remarkable symmetry in the coefficients of these functions and conjecture that all the coefficients are integers. We prove that this conjecture is true by giving an explicit construction for the weight $1 / 2$ mock theta functions, using some results found by Guerzhoy.
\end{abstract}

\section{INTRODUCTION AND STATEMENT OF THE RESULTS}

In a recent paper (see 2 2) Folsom and Ono constructed two canonical sequences of power series, using Poincaré series. One sequence consists of weight $1 / 2$ mock theta functions, the first being the Ramanujan mock theta function (of third order)

$$
f(q):=\sum_{n=0}^{\infty} \frac{q^{n^{2}}}{(1+q)^{2}\left(1+q^{2}\right)^{2} \cdots\left(1+q^{n}\right)^{2}} .
$$

In [1] and 7] we can find a description of the modularity of this function. The other sequence consists of weight $3 / 2$ weakly holomorphic modular forms. Folsom and Ono show a remarkable symmetry property of the coefficients of the functions in these sequences. They also conjecture that all coefficients are integers.

In 44 Guerzhoy shows that the coefficients are all rational. He also gives a bound on the denominator. Here we go one step further and show

Theorem 1. Let $m \leq 0$ be an integer and let

$$
H_{0, m}^{+}(\tau)=q^{-1 / 24}\left(q^{m}+\sum_{n=0}^{\infty} a_{m}(n) q^{n}\right)
$$

be the q-expansion of the mock theta function $H_{0, m}^{+}$as in [4]. Then all the numbers $a_{m}(n)$ are integers.

Remark 2. The $H_{0, m}^{+}$are the holomorphic parts of the weight $1 / 2$ Poincaré series constructed by Folsom and Ono.

For convenience we will adopt the notation used by Guerzhoy. His idea is to construct certain weakly holomorphic modular forms $F_{m}$. Combining his Propositions 1 and 3 , we get properties for these $F_{m}$ that turn out to determine them completely.

Received by the editors June 30, 2008, and, in revised form, July 2, 2008.

2000 Mathematics Subject Classification. Primary 11F11, 11F27.

(C) 2008 American Mathematical Society Reverts to public domain 28 years from publication 
Proposition 3. Let $m \leq 0$ be an integer. Then $F_{m}$ is the unique vector-valued weakly holomorphic modular form of weight $1 / 2$, which transforms as

$$
\begin{aligned}
& F_{m}(\tau+1)=\left(\begin{array}{ccc}
\zeta_{24}^{-1} & 0 & 0 \\
0 & 0 & \zeta_{3} \\
0 & \zeta_{3} & 0
\end{array}\right) F_{m}(\tau), \\
& F_{m}(-1 / \tau)=\sqrt{-i \tau}\left(\begin{array}{ccc}
0 & 1 & 0 \\
1 & 0 & 0 \\
0 & 0 & -1
\end{array}\right) F_{m}(\tau),
\end{aligned}
$$

and has the expansion

$$
F_{m}(\tau)=\left(\begin{array}{c}
q^{m-1 / 24}+\mathcal{O}\left(q^{-1 / 24}\right) \\
\mathcal{O}\left(q^{1 / 3}\right) \\
\mathcal{O}\left(q^{1 / 3}\right)
\end{array}\right) .
$$

We construct in a direct way, using an explicit basis for certain spaces of modular forms, functions with the same properties. From the construction it is immediately clear that these functions have integer coefficients. This implies that all coefficients in the Folsom-Ono grid are integers.

In Section 2 we'll give some results on holomorphic modular forms on $\Gamma_{0}(2)$. In Section 3 we'll then prove Theorem 1 and Proposition 3 ,

\section{Modular forms on $\Gamma_{0}(2)$}

Note that $\Gamma_{0}(2)$ is the subgroup of $\mathrm{SL}_{2}(\mathbb{Z})$ generated by the elements $\left(\begin{array}{ll}1 & 1 \\ 0 & 1\end{array}\right)$ and $\left(\begin{array}{cc}1 & 0 \\ -2 & 1\end{array}\right)=-\left(\begin{array}{cc}0 & -1 \\ 1 & 0\end{array}\right)\left(\begin{array}{ll}1 & 1 \\ 0 & 1\end{array}\right)^{2}\left(\begin{array}{cc}0 & -1 \\ 1 & 0\end{array}\right)$. So to show that a function $f$ is a modular form on $\Gamma_{0}(2)$, we only have to check that $f$ is 1-periodic and that $\left.f\right|_{k} S=\tau^{-k} f(-1 / \tau)$ is 2-periodic.

Definition 4. We define

$$
\Theta(\tau)=\left(\begin{array}{c}
\Theta_{0}(\tau) \\
\Theta_{1}(\tau) \\
\Theta_{2}(\tau)
\end{array}\right):=\left(\begin{array}{c}
\vartheta_{10}^{4}(0, \tau) \\
\vartheta_{01}^{4}(0, \tau) \\
\vartheta_{00}^{4}(0, \tau)
\end{array}\right),
$$

where we use the notation from [5, p. 17] for the classical Jacobi theta functions

$$
\begin{aligned}
& \vartheta_{10}(z, \tau)=\sum_{n \in 1 / 2+\mathbb{Z}} q^{n^{2} / 2} w^{n}, \\
& \vartheta_{01}(z, \tau)=\sum_{n \in \mathbb{Z}}(-1)^{n} q^{n^{2} / 2} w^{n}, \\
& \vartheta_{00}(z, \tau)=\sum_{n \in \mathbb{Z}} q^{n^{2} / 2} w^{n},
\end{aligned}
$$

with $q=\exp (2 \pi i \tau)$ and $w=\exp (2 \pi i z)$. Also we define $Z:=\Theta_{0}^{2} / 256$.

Remark 5. These functions are not independent, since they satisfy $\Theta_{0}+\Theta_{1}=\Theta_{2}$, but we don't need independence here.

Using these theta functions we can construct a basis for the space of modular forms on $\Gamma_{0}(2)$. 
Proposition 6. Let $k \geq 0$ be an integer; then $\left\{A_{k, l} \mid 0 \leq l \leq k\right\}$ is a basis for $M_{4 k+2}\left(\Gamma_{0}(2)\right)$, where

$$
A_{k, l}:=Z^{l}\left(\Theta_{1} \Theta_{2}\right)^{k-l}\left(\Theta_{1}+\Theta_{2}\right) / 2 \quad \text { for } 0 \leq l \leq k .
$$

These $A_{k, l}$ have integer coefficients and

$$
A_{k, l}=q^{l}+\mathcal{O}\left(q^{l+1}\right) .
$$

If $f \in M_{4 k+2}\left(\Gamma_{0}(2)\right)$ satisfies $\left.f\right|_{4 k+2} S=\mathcal{O}\left(q^{1 / 2}\right)$, then $f$ is a linear combination of $A_{k, l}$ with $0 \leq l \leq k-1$.

Remark 7 . We only give a basis for weight $4 k+2$, since this suffices for the proof of Theorem 1,

Remark 8. The key properties of this basis are equation (2.1) and the fact that the $A_{k, l}$ have integer coefficients. Together these properties imply that if the first $k+1$ coefficients of $f \in M_{4 k+2}\left(\Gamma_{0}(2)\right)$ are integers, then all coefficients of $f$ are integers. In the case that $\left.f\right|_{4 k+2} S=\mathcal{O}\left(q^{1 / 2}\right)$ it's even sufficient for the first $k$ coefficients to be integers.

Proof. From the classical dimension formula $\operatorname{dim}\left(M_{k}\left(\Gamma_{0}(2)\right)\right)=\lfloor k / 4\rfloor+1$ for $k$ even, we see that we indeed have the correct number of basis elements. Using table $\mathrm{V}$ in [5, p. 36] we see that

$$
\Theta(\tau+1)=\left(\begin{array}{ccc}
-1 & 0 & 0 \\
0 & 0 & 1 \\
0 & 1 & 0
\end{array}\right) \Theta(\tau), \quad \Theta(-1 / \tau)=-\tau^{2}\left(\begin{array}{lll}
0 & 1 & 0 \\
1 & 0 & 0 \\
0 & 0 & 1
\end{array}\right) \Theta(\tau),
$$

and we can easily check the expansion

$$
\Theta(\tau)=\left(\begin{array}{c}
16 q^{1 / 2}+\mathcal{O}\left(q^{3 / 2}\right) \\
1+\mathcal{O}\left(q^{1 / 2}\right) \\
1+\mathcal{O}\left(q^{1 / 2}\right)
\end{array}\right)
$$

Using these properties, it is easy to see that $\left(\Theta_{1}+\Theta_{2}\right) / 2, \Theta_{1} \Theta_{2}$ and $Z$ are holomorphic modular forms with integer coefficients of weight 2, 4 and 4 respectively on $\Gamma_{0}(2)$ and that

$$
\begin{aligned}
\left(\Theta_{1}+\Theta_{2}\right) / 2 & =1+\mathcal{O}(q), \\
\Theta_{1} \Theta_{2} & =1+\mathcal{O}(q), \\
Z & =q+\mathcal{O}\left(q^{2}\right) .
\end{aligned}
$$

From this we get that the $A_{k, l}$ are indeed modular forms of weight $4 k+2$ on $\Gamma_{0}(2)$, with integer coefficients, that satisfy equation (2.1). From this equation we immediately see that the $A_{k, l}$ are linearly independent. From the identity

$$
\left.A_{k, l}\right|_{4 k+2} S=-\left(\Theta_{1}^{2} / 256\right)^{l}\left(\Theta_{0} \Theta_{2}\right)^{k-l}\left(\Theta_{0}+\Theta_{2}\right) / 2,
$$

we see that

$$
\left.A_{k, l}\right|_{4 k+2} S=\mathcal{O}\left(q^{(k-l) / 2}\right),
$$

from which it follows that if $\left.f\right|_{4 k+2} S=\mathcal{O}\left(q^{1 / 2}\right)$, then the coefficient of $A_{k, k}$ is zero. 


\section{Proofs of Theorem 1 and Proposition 3}

The transformation properties and the expansion in Proposition 3 follow directly from Propositions 1 and 3 of [4. To prove the uniqueness, we consider the following theta functions of weight $3 / 2$, as in [4] and [7]:

$$
\begin{aligned}
& g_{0}(\tau)=\sum_{n \in \mathbb{Z}}(-1)^{n}(n+1 / 3) e^{3 \pi i(n+1 / 3)^{2} \tau} \\
& g_{1}(\tau)=-\sum_{n \in \mathbb{Z}}(n+1 / 6) e^{3 \pi i(n+1 / 6)^{2} \tau} \\
& g_{2}(\tau)=\sum_{n \in \mathbb{Z}}(n+1 / 3) e^{3 \pi i(n+1 / 3)^{2} \tau} .
\end{aligned}
$$

These theta functions have the modular transformation properties

$$
\left(\begin{array}{c}
g_{1} \\
g_{0} \\
-g_{2}
\end{array}\right)(\tau+1)=\left(\begin{array}{ccc}
\zeta_{24} & 0 & 0 \\
0 & 0 & \zeta_{3}^{-1} \\
0 & \zeta_{3}^{-1} & 0
\end{array}\right)\left(\begin{array}{c}
g_{1} \\
g_{0} \\
-g_{2}
\end{array}\right)(\tau)
$$

and

$$
\left(\begin{array}{c}
g_{1} \\
g_{0} \\
-g_{2}
\end{array}\right)(-1 / \tau)=-(-i \tau)^{3 / 2}\left(\begin{array}{ccc}
0 & 1 & 0 \\
1 & 0 & 0 \\
0 & 0 & -1
\end{array}\right)\left(\begin{array}{c}
g_{1} \\
g_{0} \\
-g_{2}
\end{array}\right)(\tau)
$$

If we now define

$$
\Psi_{m}:=\left(\begin{array}{c}
g_{1} F_{0, m} \\
g_{0} F_{1, m} \\
-g_{2} F_{2, m}
\end{array}\right)
$$

then we get from the transformation properties and the expansion of $F_{m}$ that $\Psi_{m}$ transforms as

$$
\Psi_{m}(\tau+1)=\left(\begin{array}{ccc}
1 & 0 & 0 \\
0 & 0 & 1 \\
0 & 1 & 0
\end{array}\right) \Psi_{m}(\tau), \quad \Psi_{m}(-1 / \tau)=\tau^{2}\left(\begin{array}{ccc}
0 & 1 & 0 \\
1 & 0 & 0 \\
0 & 0 & 1
\end{array}\right) \Psi_{m}(\tau)
$$

and has the expansion

$$
\Psi_{m}(\tau)=\left(\begin{array}{c}
-1 / 6 q^{m}+\mathcal{O}(1) \\
\mathcal{O}\left(q^{1 / 2}\right) \\
\mathcal{O}\left(q^{1 / 2}\right)
\end{array}\right)
$$

If we have two functions $\widehat{\Psi}_{m}$ and $\tilde{\Psi}_{m}$ that have these transformation properties and both have an expansion as in equation (3.2), then their difference again has the transformation properties as in equation (3.1) and has the expansion

$$
\left(\widehat{\Psi}_{m}-\tilde{\Psi}_{m}\right)(\tau)=\left(\begin{array}{c}
\mathcal{O}(1) \\
\mathcal{O}\left(q^{1 / 2}\right) \\
\mathcal{O}\left(q^{1 / 2}\right)
\end{array}\right) .
$$

If we take the product of the three components of $\widehat{\Psi}_{m}-\tilde{\Psi}_{m}$, then we get a holomorphic modular form of weight 6 on $\mathrm{SL}_{2}(\mathbb{Z})$, which is $\mathcal{O}(q)$ and hence is zero. By equation (3.1) this implies that all three components of $\widehat{\Psi}_{m}-\tilde{\Psi}_{m}$ are zero and so $\Psi_{m}$ is the unique vector-valued weakly holomorphic modular form satisfying equations (3.1) and (3.2). By definition of $\Psi_{m}$, this implies the uniqueness in Proposition 3 . 
From equation (3.1) we see that $g_{1} F_{0, m}$, which is the first component of $\Psi_{m}$, is a weakly holomorphic modular form of weight 2 on $\Gamma_{0}(2)$, and hence $P_{m}:=$ $-6 Z^{-m} g_{1} F_{0, m}$ is a holomorphic modular form of weight $-4 m+2$ on $\Gamma_{0}(2)$. From the expansion of $F_{m}$ given in Proposition 3 , we see that the first $-m$ coefficients in the Fourier series of $P_{m}$ are the same as those of $-6 q^{m-1 / 24} Z^{-m} g_{1}$, which are integers, and that $\left(\left.P_{m}\right|_{-4 m+2} S\right)(\tau)=\mathcal{O}\left(q^{1 / 2}\right)$. If we combine this observation with Proposition 6. we see that we can write $P_{m}$ as a linear combination of $A_{-m, l}$ with $0 \leq l \leq-m-1$, where the coefficients are integers which are determined by the first $-m$ coefficients in the Fourier expansion of $-6 q^{m-1 / 24} Z^{-m} g_{1}$. Hence the $F_{m}$ have integer coefficients and combined with Proposition 3 in 4 and the fact that the holomorphic part of $H_{0,0}$, which is the Ramanujan mock theta function $f$ given in the introduction, has integer coefficients, we get that all the coefficients in the Folsom-Ono grid are integers.

Note that this construction allows us to determine $F_{m}$ explicitly for a given $m$; for example, using PARI/GP (see [6]) we found

$$
\begin{aligned}
F_{-1} & =-\frac{\Theta_{1} \Theta_{2}\left(\Theta_{1}+\Theta_{2}\right)}{12 Z g_{1}} \\
& =q^{-1 / 24}\left(q^{-1}+5-258 q+2771 q^{2}-17945 q^{3}+87497 q^{4}-\ldots\right) \\
F_{-2} & =-\frac{\Theta_{1} \Theta_{2}\left(\Theta_{1}+\Theta_{2}\right)}{12 Z^{2} g_{1}}\left(\Theta_{1} \Theta_{2}+19 Z\right) \\
& =q^{-1 / 24}\left(q^{-2}-7+3393 q-102046 q^{2}+1477260 q^{3}-14374130 q^{4}+\ldots\right) \\
F_{-3} & =-\frac{\Theta_{1} \Theta_{2}\left(\Theta_{1}+\Theta_{2}\right)}{12 Z^{3} g_{1}}\left(\Theta_{1}^{2} \Theta_{2}^{2}+43 \Theta_{1} \Theta_{2} Z+187 Z^{2}\right) \\
& =q^{-1 / 24}\left(q^{-3}-23374 q+1633513 q^{2}-44554875 q^{3}+738914605 q^{4}-\ldots\right) .
\end{aligned}
$$

These coefficients are indeed consistent with the ones given in the corrigendum (see 3]) to [2], where a corrected table of coefficients is provided. To compare the coefficients, note that from Proposition 3 of [4] we have $F_{-1}=q^{-1 / 24}\left(q^{-1}-1+\right.$ $\left.f_{2}+5 f_{1}\right), F_{-2}=q^{-1 / 24}\left(q^{-2}-1+f_{3}-7 f_{1}\right)$, etc.

In general we have $F_{m}=-\frac{\Theta_{1}+\Theta_{2}}{12 g_{1}} r_{m}\left(\Theta_{1} \Theta_{2} / Z\right)$, where $r_{m}$ is a polynomial of degree $-m$, the first few being

$$
\begin{aligned}
& r_{-1}(x)=x \\
& r_{-2}(x)=x^{2}+19 x \\
& r_{-3}(x)=x^{3}+43 x^{2}+187 x \\
& r_{-4}(x)=x^{4}+67 x^{3}+943 x^{2}+1292 x \\
& r_{-5}(x)=x^{5}+91 x^{4}+2275 x^{3}+14104 x^{2}+7106 x \\
& r_{-6}(x)=x^{6}+115 x^{5}+4183 x^{4}+52260 x^{3}+162196 x^{2}+33231 x \\
& r_{-7}(x)=x^{7}+139 x^{6}+6667 x^{5}+129584 x^{4}+914550 x^{3}+1531961 x^{2}+137394 x \\
& r_{-8}(x)=x^{8}+163 x^{7}+9727 x^{6}+259900 x^{5}+3045224 x^{4}+13015219 x^{3} \\
& \quad+12389380 x^{2}+515185 x .
\end{aligned}
$$




\section{ACKNOWLEDGEMENTS}

The author thanks Pavel Guerzhoy for helpful discussions and Ken Ono and Amanda Folsom for explaining the initial discrepancy between the coefficients they found and the ones given here.

\section{REFERENCES}

[1] K. Bringmann and K. Ono, The $f(q)$ mock theta function conjecture and partition ranks, Invent. Math. 165 (2006), pp. 243-266. MR2231957 (2007e:11127)

[2] A. Folsom and K. Ono, Duality involving the mock theta function $f(q)$, J. London Math. Soc. 77 (2008), pp. 320-334. MR.2400394

[3] _ Corrigendum: Duality involving the mock theta function $f(q)$, J. London Math. Soc., to appear.

[4] P. Guerzhoy, Rationality of the Folsom-Ono grid, Proc. Amer. Math. Soc., accepted for publication.

[5] D. Mumford, Tata Lectures on Theta. I, Progress in Mathematics, no. 28, Birkhäuser Boston, 1983. MR688651 (85h:14026)

[6] PARI/GP, version 2.3.1, Bordeaux, 2006, http://pari.math.u-bordeaux.fr/.

[7] S. P. Zwegers, Mock $\theta$-functions and real analytic modular forms, $q$-Series with Applications to Combinatorics, Number Theory, and Physics, Contemporary Mathematics, vol. 291, Amer. Math. Soc., 2001, pp. 269-277. MR1874536 (2003f:11061)

School of Mathematical Sciences, University College Dublin, Belfield, Dublin 4, IRELAND

E-mail address: sander.zwegers@ucd.ie 